\title{
A Semantic Characterization of Locative PPs
}

\author{
Joost Zwarts Yoad Winter \\ Utrecht Institute of Linguistics OTS
}

\section{Introduction}

The last two decades have seen remarkable development in compositional modeltheoretic semantics of natural language. The idea that natural language expressions can be directly interpreted in the model of discourse has gained significant support in various domains. It led to a better understanding of inference in linguistics with close relation to syntax, helped to characterize linguistically relevant classes of expressions, and revealed systematic constraints on their possible meanings. Quite independently of these developments, much work in the fields of cognitive and conceptual semantics has acquired a significant body of knowledge about the semantic behaviour of prepositional phrases (PPs). In the modeltheoretic framework, however, the semantics of PPs has remained, to a large extent, unexplored. Our aim in this paper is to contribute for bridging this gap.

We refine and extend the proposal in Zwarts (1995), arguing for a vector space as the underlying ontology in the compositional analysis of locative PP structures. In section 2 we introduce a general semantic framework that uses such a model. Section 3 studies some denotational properties of prepositions in the proposed system. Certain properties introduced in Zwarts (1995) will be placed here in a wider perspective of preposition monotonicity. Two notions of monotonicity are defined and shown to be linguistically relevant in restricting the set of possible preposition denotations, accounting for central inferences and affecting grammaticality of modified PPs. An additional constraint, similar to the conservativity restriction on determiners, is shown to hold of natural language locative prepositions.

Throughout this paper, we presuppose familiarity with basic Linear Algebra and simple notions from Topology. ${ }^{1}$ Some useful definitions are summarized in an appendix.

\section{Vector Semantics of Locative PPs}

Sentences with spatial uses of prepositions show inferential regularities that are comparable with the much studied inferences with quantified expressions. For instance, like the determiner every, the preposition inside is transitive, in the sense illustrated in (1). The preposition near is symmetric similar to the determiner some, as exemplified in (2). ${ }^{2}$

\begin{tabular}{|c|c|c|c|c|c|}
\hline \multirow{2}{*}{ (1) } & $\mathrm{A}$ is inside $\mathrm{B}$ & every $\mathrm{A}$ is $\mathrm{B}$ & \multirow[b]{2}{*}{ (2) } & \multirow{2}{*}{$\begin{array}{l}A \text { is near } B \\
B \text { is near } A\end{array}$} & \multirow{2}{*}{$\begin{array}{l}\text { some } A \text { is } B \\
\text { some } B \text { is } A\end{array}$} \\
\hline & $\mathrm{B}$ is inside $\mathrm{C}$ & every $\mathrm{B}$ is $\mathrm{C}$ & & & \\
\hline
\end{tabular}

As far as determiners concern, this kind of observations about inferences is the 
empirical basis for the generalized quantifier semantics of the noun phrase. That prepositions show similar consistencies is a reason to develop also a modeltheoretic semantics of the prepositional phrase, with a similar research agenda to the one of generalized quantifier theory (see e.g. Keenan (1996)).

\subsection{A preliminary typology of spatial prepositions}

The preposition is the most useful syntactic category in natural languages for expressing statements about space and movement. ${ }^{3}$ Locative prepositions are used to locate an object relative to another one, the reference object. For instance, in the predicative constructions $(3 \mathrm{a} / \mathrm{b})$ the house is the reference object and the tree is the located object. Directional prepositions are more "dynamic" than the locative ones: they are usually connected to a verb or a noun expressing movement or direction as in (4a). Unlike the locative ones, the directional prepositions often resist predicative constructions (cf. (4b)).

(3) a. The tree is outside the house.

b. The tree is behind the house.

(4) a. John walked to the park.

b. ?John is to the park.

We concentrate on the locative prepositions and PPs, which can be classified into projective and non-projective. A non-projective preposition like outside in (3a) requires only spatial knowledge on the location of the two objects. By contrast, the projective preposition behind requires further information about directions from the reference object. To determine whether (3b) is true, the shape and location of the tree and the house are not sufficient. Also the back side of the house should be determined. This can change with the position of the speaker or conventions of using the house and hence it is not only a function of its intrinsic spatial properties. These distinctions (cf. Herskovits (1986)) are further exemplified in (5)-(6).

(5) Locative prepositions:

a. Projective: above, below, in front of, behind, beside

b. Non-projective: in/inside, outside, on, at, near, between

(6) Directional prepositions: to, from, into, onto, across, around, through

\subsection{The modification problem}

Many locative PPs can be modified by expressions that involve some measure of distance or direction. For example, in addition to the "bare" PP structures of (3), there are also modified structures as in (7). Some other cases of PP modification are exemplified in (8).

(7) a. The tree is ten meters [outside the house].

b. The tree is ten meters [behind the house].

(8) diagonally above the door, far outside the city, right in front of the car 
These structures are classified as PP modification because the additional expression syntactically applies to a PP (or a P-bar) to produce another PP (P-bar). Zwarts (1995) discusses the problem of giving a compositional semantics to such structures. Previous works (e.g. Wunderlich (1991) a.o.) propose to treat prepositions as relations between sets of points (regions). A region $A$ is outside a region $B$ iff the two regions are disjoint. Compositionally, outside denotes a function mapping a region $A$ to the set of regions disjoint to it: outside $(A)=\{X: X \cap A=\emptyset\}$. This predicate over regions compositionally applies to $B: B \in$ outside $(A)$. Suppose now that in (3a) and in (7a) the house occupies a region $A$ and the tree occupies a region $B$ consisting of a single point $p$. The analysis of (3a) is straightforward and tantamount to $p \notin A$. In order to analyze compositionally also ( $7 \mathrm{a}$ ), the denotation of the modifier ten meters should apply to the denotation of outside $(A)$. To get the correct semantics, this function has to measure the distance between $p$ and $A$. But this is problematic, as $A$ is not directly specified in the set outside $(A)$. One may try to approach the problem by reproducing $A$ from this set (e.g. observing that $A$ is the complement of $\cup$ outside $(A)$ ). However, the same ad hoc procedure would not correctly hold of (7b). A general compositional treatment of PP modification is not forthcoming if locative prepositions are taken as relations between sets of points.

Zwarts (1995) makes the following observations. Modifiers like ten meters and diagonally are predicates over distance and direction respectively. Hence also the function that a locative preposition denotes should return entities with measurable distance and direction. These entities are proposed to be vectors: directed line segments between points in space. Assume that an expression like outside the house denotes a set of vectors: roughly, the ones pointing outwards from the boundary of the house. Cases of syntactic modification as in (7)-(8) are naturally analyzed as (intersective) semantic modification: a PP modifier is a function that maps any set of vectors to one of its subsets. For instance, ten meters maps a set of vectors $W$ to its subset containing only vectors that are ten meter long: $\{v \in W:|v|=10 \mathrm{~m}\}$. Thus, ten meters outside the house denotes the set of ten meter vectors pointing outwards from the house. A locative preposition then denotes a function that applies to the set of points where the reference object is located and returns a set of vectors. The next section substantiates this proposal. ${ }^{4}$

\subsection{Vector space ontology}

A natural way to implement the proposal in Zwarts (1995) is to assume that vectors are the primitive spatial entity in models of natural language. ${ }^{5}$ Space ontology consists of a vector space $V$ over the real numbers $\mathbf{R}$. The element $0 \in V$ is the zero vector and the functions $+:(V \times V) \rightarrow V$ and $\cdot:(\mathbf{R} \times V) \rightarrow V$ are vector addition and scalar multiplication respectively. We assume a positive scalar product $f:(V \times V) \rightarrow \mathbf{R}^{+}$, standardly defining a norm ||$: V \rightarrow \mathbf{R}^{+}$. It is further assumed that $V$ is an Euclidean $n$-space $\mathbf{R}^{n}$. Drawing on this ontology, we define the domain of points $D_{p}$ and the domain of $v$ ectors $D_{v}$. $D_{p}$ is simply identified with $V$. Intuitively, each vector in $V$ uniquely determines its end-point and vice versa. The domain $D_{v}$ is defined as the cartesian product $V \times V$. Each "point" $w$ in $D_{p}$ 
(= a vector in $V$ ) functions as "the center" (= the zero vector) of a vector space $V_{w} \subseteq D_{v}$. This is done as in the following definition.

Definition 1 (the vector domain) Let $\langle V, 0,+, \cdot\rangle$ be a vector space over $\mathbf{R}$ with $f$ a positive scalar product and $w \in V$. We define:

$$
\begin{aligned}
& V_{w} \stackrel{\text { def }}{=}\{\langle w, v\rangle: v \in V\} \\
& 0_{w} \stackrel{\text { def }}{=}\langle w, 0\rangle \\
& \text { For all } u, v \in V:\langle w, u\rangle+{ }_{w}\langle w, v\rangle \stackrel{\text { def }}{=}\langle w, u+v\rangle \\
& \text { For all } s \in \mathbf{R}, v \in V: s \cdot{ }_{w}\langle w, v\rangle \stackrel{\text { def }}{=}\langle w, s \cdot v\rangle \\
& \text { For all } u, v \in V: f_{w}(\langle w, u\rangle,\langle w, v\rangle) \stackrel{\text { def }}{=} f(u, v)
\end{aligned}
$$

For every $w \in V:\left\langle V_{w}, 0_{w},{ }{ }_{w},{ }_{w}\right\rangle$ is a vector space over $\mathbf{R}$ with $f_{w}$ a positive scalar product, which determines a norm denoted by ||$_{w}$. Trivially, the domain $D_{v}$ is equal to the union of vector spaces $\cup_{w \in V} V_{w}$.

Notational conventions (see fig. la): $\mathbf{p}, \mathbf{q} \in D_{p}$ for points; $\mathbf{u}, \mathbf{v}, \mathbf{w} \in D_{v}$ for vectors; if $\mathbf{u}=\langle w, v\rangle \in D_{v}$ then $s$-point $(\mathbf{u}) \stackrel{\text { def }}{=} w \in V$ is the start-point of $\mathbf{u}$, e-point $(\mathbf{u}) \stackrel{\text { def }}{=} u+v \in V$ is its end-point. The vectors $w$ and $w+v$ can be viewed as "points" $\mathbf{p}$ and $\mathbf{q}$ in $D_{p}$. We sloppily use the symbols $+, \cdot, f$ and || for operators on members of $V_{w}$, without mentioning the subscript $w$ as strictly required. $D_{p}$ and $D_{v}$ are treated as typed domains of types $p$ and $v$ respectively.

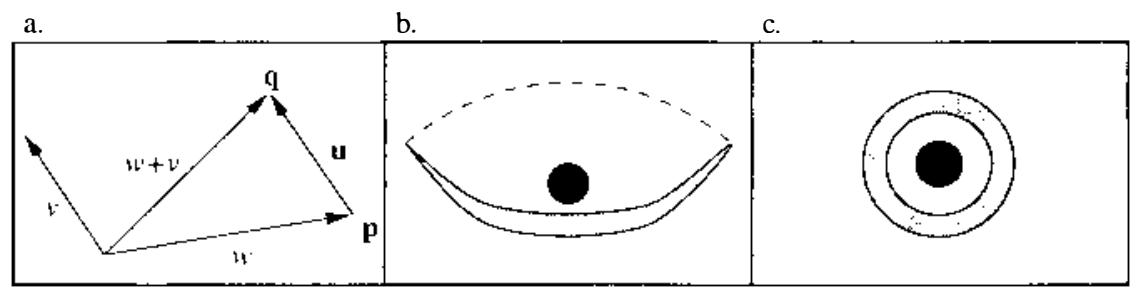

Figure 1:

\subsection{The compositional process}

Reconsider the modified structures in (7). The proposed "semantic structure" of a modified PP with a modifier MOD, a preposition $\mathrm{P}$ and a reference object region REF is as follows:

$$
\operatorname{MOD}_{(v t)(v t)}\left(\mathrm{P}_{(p t)(v t)}\left(\mathrm{REF}_{p t}\right)\right)
$$

The assumed denotation of a measure phrase modifier MOD is straightforward (unlike the analysis of its compositional semantics). For instance:

ten_meters $\stackrel{\text { def }}{=} \lambda W_{v t} \cdot \lambda \mathbf{v} . W(\mathbf{v}) \wedge|\mathbf{v}|=10 \mathrm{~m}$

The constant $m$ (for meter) is a positive real number with the familiar fixed relation to other similar measure constants (e.g. for feet). 
The denotations of various locative prepositions for $\mathrm{P}$ will be defined in the next section. The region REF is determined by the denotation of the $e$-type reference object. A location function $l o c_{e}(p t)$ assigns any physical entity in $D_{e}$ its location in space, or eigenspace (Wunderlich (1991)). Since a PP basically denotes a set of vectors, we have to translate it into an "ordinary" et predicate that standardly applies to the subject of predication (the located object). An "anti-location" function $\mathrm{loc}^{-}$returns the objects at the region determined by the set of vectors:

$$
l o c \stackrel{\text { def }}{=} \lambda W_{v t} \cdot \lambda x_{e} \cdot \forall \mathbf{p} \in \operatorname{loc}(x) \exists \mathbf{v} \in W[e-p o i n t(\mathbf{v})=\mathbf{p}]
$$

In words: $l o c^{-}$maps any set of vectors $W$ to the set of entities whose eigenspace is contained in the set of $W$ 's end-points.

To exemplify the process, sentence (7a) denotes the following proposition.

(9) $l o c^{-}\left(\right.$ten_meters $^{\prime}\left(\right.$ outside $^{\prime}\left(\operatorname{loc}\left(\right.\right.$ the_house $\left.\left.\left.\left.^{\prime}\right)\right)\right)\right)\left(\right.$ the_tree $\left.^{\prime}\right) \Leftrightarrow$

$\forall \mathbf{p} \in \operatorname{loc}\left(\right.$ the_tree $\left.^{\prime}\right) \exists \mathbf{v} \in$ outside' $^{\prime}\left(\operatorname{loc}\left(\right.\right.$ the_house $\left.\left.^{\prime}\right)\right)[\mathbf{p}=e$-point $(\mathbf{v}) \wedge$ $|\mathbf{v}|=10 \mathrm{~m}]$

Proposition (9) claims that every point in the tree is an end-point of a $10 \mathrm{~m}$ long vector starting on the house and pointing outside. ${ }^{6}$ To complete the picture, we have to give the definition of preposition denotations like outside'.

\subsection{Denotations of locative prepositions}

Preposition meanings do not fully exploit the possibilities that mathematical theories of space allow. One example is convexity. Although speakers may well be aware that some object is not physically convex, there is a tendency to ignore this fact and to conceptually "convexize" the object. For instance, although the bowl in figure $1 \mathrm{~b}$ occupies a non-convex region, disjoint from the space occupied by the ball, this situation can nevertheless be described by the sentence the ball is inside the bowl. The bowl is conceived of as if it were a convex object, roughly of the shape indicated by the dashed line, which does contain the ball. Similarly, it is quite strange to say that in figure lc the black circle is outside the grey ring. Rather, inside is more appropriate here. Treating such effects would lead us too far afield (see Herskovits (1986)). Instead, we tentatively assume that the loc function maps entities only to convex eigenspaces. For similar reasons we assume that eigenspaces of objects are closed and non-trivial (= non-empty and properly contained in $D_{p}$ ). Regions that have these three properties are referred to as Topologically Simple. We speculate that cases as in fig. 1b-c should be handled by a theory of visual perception and not necessarily by linguistic semantics. Such a theory should provide details about the cognitive parameters in the determination of the loc function.

The semantic task we are facing is to define $(p t)(v t)$ functions for locative prepositions that respect basic inferences in natural language given the syntactic, compositional and ontological assumptions above. The following definitions are helpful in the treatment of many prepositions.

Definition 2 (external/internal vectors) Let $\mathbf{v} \in D_{v}$ be a vector and $A \subseteq D_{p} a$ set of points s.t. s-point $(\mathbf{v})$ is in $b(A)$, the boundary of $A$. The vector $\mathbf{v}$ is called external to $A$ if e-point $(\mathbf{v}) \in D_{p} \backslash A$, and internal to $A$ if e-point $(\mathbf{v}) \in A$. 


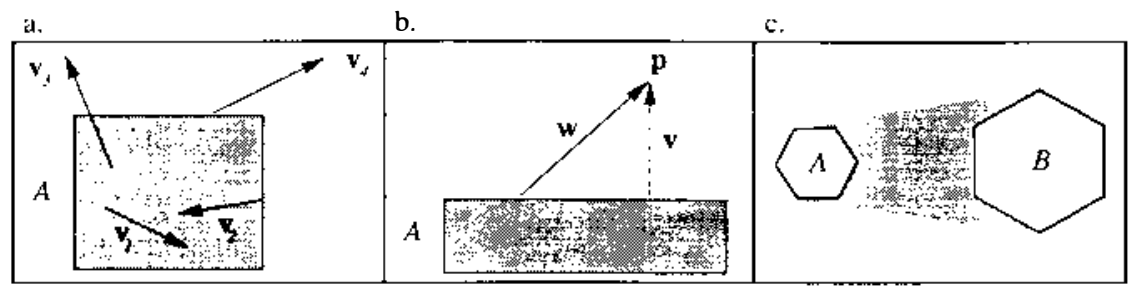

Figure 2:

For example, in figure $2 \mathrm{a}: \mathbf{v}_{4}$ is external to $A, \mathbf{v}_{2}$ is internal to $A$, and $\mathbf{v}_{1}$ and $\mathbf{v}_{3}$ are neither internal nor external to $A$. Zero vectors in the boundary of $A$ are considered internal to $A$.

Definition 3 (externally/internally closest vectors) $A$ vector $\mathbf{v} \in D_{v}$ is externally (internally) closest to a set of points $A \subseteq D_{p}$ iff $\mathbf{v}$ is external (internal) to $A$ and for every vector $\mathbf{w} \in D_{v}$ that is external (internal) to $A$ s.t. e-point $(\mathbf{v})=e$-point $(\mathbf{w})$ : $|\mathbf{v}| \leq|\mathbf{w}|$. In case this holds we denote ext $(\mathbf{v}, A)(\operatorname{int}(\mathbf{v}, A))$.

Definition 3 imposes a condition of minimality: it classifies vectors external/internal to a set of points $A$ that are the shortest vectors connecting points in $A$ 's boundary to points outside/inside $A$. This condition is required because of the semantics of $\mathrm{PP}$ modification. For example, a point $\mathrm{p}$ as in figure $2 \mathrm{~b}$ can be said to be exactly three meters outside the box $A$ only if the shortest vector connecting it to the box, $\mathbf{v}$, is three meters long. Longer connections like $\mathbf{w}$ are irrelevant. In a similar way, $\mathrm{p}$ is not diagonally above the box although $\mathrm{w}$ is a vector diagonal to the box. Correspondingly, while the vector $\mathbf{v}$ is defined as externally closest to $A, \mathbf{w}$ is not.

Under the topological simplicity assumption these notions are related to the intuitive definition of external/internal points using set membership. Consider first the following topological property (see Wall (1972:p.18)):

Proposition 1 If $A$ and $B$ are disjoint closed subsets of $\mathbf{R}^{n}$ and $A$ is compact, then $\operatorname{dist}(A, B)$, the distance between $A$ and $B$, which is defined by the infimum $\inf (\{\operatorname{dist}(a, b): a \in A, b \in B\})$, is positive.

By this property, we can show the following correspondence:

Proposition 2 Let $A \subset D_{p}$ be a non-trivial closed set in $D_{p}\left(=\mathbf{R}^{n}\right)$. Then for every point $\mathbf{p} \in D_{p}$ the following conditions are equivalent: (a) There is a vector $\mathbf{v} \in D_{v}$ that is externally (internally) closest to $A$ s.t. e-point $(\mathbf{v})=\mathbf{p} . \quad(\mathbf{b}) \mathbf{p} \notin A(\mathbf{p} \in A)$

Proof: $(a) \Rightarrow(b)$ directly by the definition of external/internal vectors. Let us show $(b) \Rightarrow(a)$.

1. Assume $\mathbf{p} \notin A$. $\{\mathbf{p}\}$ is bounded and closed in $\mathbf{R}^{n}$, hence compact. Thus by proposition $1, \operatorname{dist}(\mathbf{p}, A)>0$. Let $C$ be a closed sphere around $\mathbf{p}$ of radius $r=\operatorname{dist}(\mathbf{p}, A)$.

Let us show $C \cap A \neq \emptyset$. Assume by negation $C \cap A=\emptyset$. By definition of $\operatorname{dist}(\mathbf{p}, A)$ : 
for every $\epsilon>0$ there is $\mathbf{q} \in A$ s.t. $r \leq \operatorname{dist}(\mathbf{p}, \mathbf{q})<r+\epsilon$. The line segment $[\mathbf{p}, \mathbf{q}]$ intersects $b(C)$, the boundary of $C$, at point $\mathbf{p}^{\prime}$. Thus, $0 \leq \operatorname{dist}\left(\mathbf{p}^{\prime}, \mathbf{q}\right)<\epsilon$. $C$ is closed, hence $b(C) \subseteq C$, so $\mathbf{p}^{\prime} \in C$.

Conclusion: $\operatorname{dist}(C, A)=\inf \left(\left\{\operatorname{dist}\left(\mathbf{p}^{\prime}, \mathbf{q}\right): \mathbf{p}^{\prime} \in C, \mathbf{q} \in A\right\}\right)=0$. But $C$ is bounded and closed in $\mathbf{R}^{n}$, hence compact. By our assumption $C \cap A=\emptyset$. Thus by proposition $1, \operatorname{dist}(C, A)>0$. Contradiction.

We conclude that $C \cap A \neq \emptyset$. It is easy to show $C \cap A \subseteq b(A)$. Thus, for any $\mathbf{q} \in$ $C \cap A$, the vector $\mathbf{v} \in D_{v}$ from $\mathbf{q}$ to $\mathbf{p}$ satisfies $r=|\mathbf{v}|=\operatorname{dist}(\mathbf{p}, \mathbf{q})=\operatorname{dist}(\mathbf{p}, A)$. Therefore, $\mathbf{v}$ is externally closest to $A$ with e-point $(\mathbf{v})=\mathbf{p}$.

2. Assume $\mathbf{p} \in A .(b) \Rightarrow(a)$ trivially holds if $\mathbf{p} \in b(A)$ : the zero vector from $\mathbf{p}$ to $\mathbf{p}$ is internally closest to $A$. For $\mathbf{p}$ is in the interior of $A, i(A)$, repeat the above prooffor $\mathbf{p}$ and $D_{p} \backslash i(A)$ (a closed set) and note that $b\left(D_{p} \backslash i(A)\right)=b(A)$.

Consider first the prepositions in/inside and outside. In our proposal they map a set of points to the set of its internally/externally closest vectors respectively. Thus, we simply define:

$$
\begin{aligned}
& \text { in, inside }{ }^{7}: \quad \text { inside } \stackrel{\text { def }}{=} \lambda A . \lambda \mathbf{v} . \operatorname{int}(\mathbf{v}, A) \\
& \text { outside: } \quad \text { outside } \stackrel{\text { def }}{=} \lambda A \cdot \lambda \mathbf{v} \cdot \operatorname{ext}(\mathbf{v}, A)
\end{aligned}
$$

The compositional procedure and proposition 2 guarantee that these definitions coincide with the intuition that inside and outside correspond to set containment and disjointness, respectively.

Corollary 3 Let the eigenspace loc(a) of an object a be a non-trivial closed set. Then the following holds: 1 . $\mathbf{b}$ is inside a is true iff $\operatorname{loc}(\mathbf{b}) \subseteq \operatorname{loc}(\mathbf{a}) \quad 2$. $\mathbf{b}$ is outside $\mathbf{a}$ is true iff $\operatorname{loc}(\mathbf{b}) \cap \operatorname{loc}(\mathbf{a})=\emptyset$.

This seemingly trivial result shows that the vector semantics of prepositions like outside, designed to deal with their allowing for PP modification, still preserves the basic set-theoretical intuition of the point semantics. The achievement of both goals is not trivial.

All prepositions except inside give rise to regions that are outside the eigenspace, so the relation $\operatorname{ext}(\mathbf{v}, X)$ is a part of their definition. The preposition imposes an additional condition on the vector $\mathbf{v}$. This condition can involve the length $\mathbf{v}$, as in the following definitions:

$$
\begin{array}{ll}
\text { on, at: } & \text { on' } \stackrel{\text { def }}{=} \lambda A \cdot \lambda \mathbf{v} \cdot \operatorname{ext}(\mathbf{v}, A) \wedge|\mathbf{v}|<r_{0} \\
\text { near: } & \text { near' } \stackrel{\text { def }}{=} \lambda A \cdot \lambda \mathbf{v} \cdot \operatorname{ext}(\mathbf{v}, A) \wedge|\mathbf{v}|<r_{1} \\
& \text { where } r_{0} \text { and } r_{1} \text { two small positive numbers } r_{0} \approx 0 \text { and } r_{0}<<r_{1}
\end{array}
$$

We interpret $o n$ and $a t$ as requiring almost zero distance between the objects. In the case of near the vector's length is said to be smaller that a pragmatically determined number $r_{1}{ }^{8}$

The prepositions between and amid require two or more reference objects. We define between' as corresponding to a non-constituent expression between... and.... A more adequate treatment requires an analysis of plurality. The function between' maps two regions $A$ and $B$ to a set of vectors using the region $\operatorname{co}(A \cup B)$, 


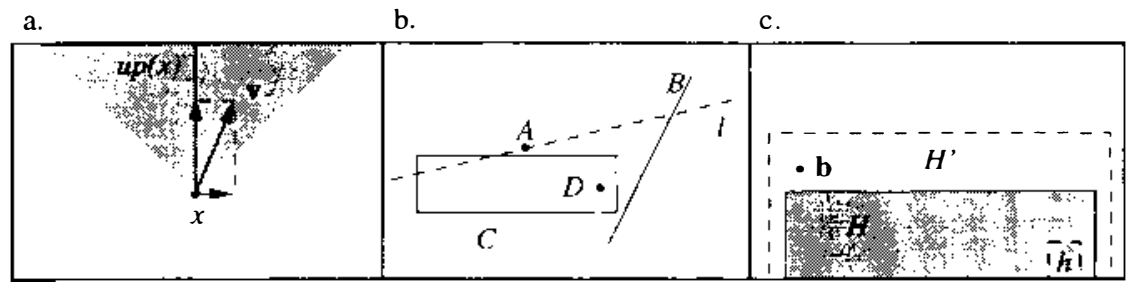

Figure 3:

the convex hull of $A \cup B$. For regions $A$ and $B$ as in figure 2c, between' $(A)(B)$ is the set of vectors that are externally closest to $A$ or to $B$ whose end-point is in the shaded region.

(12) between...and...:

between' $\stackrel{\text { def }}{=} \lambda A \cdot \lambda B . \lambda \mathbf{v} \cdot[\operatorname{ext}(\mathbf{v}, A) \vee \operatorname{ext}(\mathbf{v}, B)] \wedge e-\operatorname{point}(\mathbf{v}) \in \operatorname{co}(A \cup B)$

As mentioned, the projective prepositions presuppose certain directions in space. We assume that this is pragmatically determined using three orthogonal unit vectors in $V$ for up, right and front, which are called axes. For every start-point $w \in V$ of vectors in $V_{w} \subseteq D_{v}$, an axis $a \in V$ determines an axis $\langle w, a\rangle \in V_{w}$ that we denote $a(w)$.

Consider the projective preposition above. The region this preposition generates when the reference object is a single point $x$ is illustrated in figure $3 \mathrm{a}$. The above-region consists of those vectors that make an acute angle with the $u p(x)$ axis. Thus, above allows only vectors whose vertical component (on $u p$ ) is larger than their projection on the orthogonal component $\perp u p$ (=the horizontal plane). This is defined using the following fact from linear algebra (see e.g. Lang (1977:p.134)).

Proposition 4 For every $v, a \in V$ where $a \neq 0$ there is a unique scalar $c(a, v) \in \mathbf{R}$ and $a$ unique vector $v_{\perp a}$ s.t. $v=c(a, v) \cdot a+v_{\perp a}$. The scalar $c(a, v)$ is called $v$ 's component along $a$ and the vectors $v_{a}=c(a, v) \cdot a$ and $v_{\perp_{a}}$ are called $v$ 's projection on $a$ and on $\perp$ a respectively.

For a vector $\mathbf{v} \in V_{w}$ and an axis $a \in V$, we denote $c(a, \mathbf{v})=c(a(w), \mathbf{v}), \mathbf{v}_{a}=$ $\mathbf{v}_{a(w)}$ and $\mathbf{v}_{\perp a}=\mathbf{v}_{\perp a(w)}$. The denotations of above and below are defined in (13). Other projective prepositions as in (14) work in a similar way but with other axes.

$$
\begin{aligned}
& \text { above: } \quad \text { above' } \stackrel{\text { def }}{=} \lambda \cdot A \cdot \lambda \mathbf{v} \cdot \operatorname{ext}(\mathbf{v}, A) \wedge c(u p, \mathbf{v})>\left|\mathbf{v}_{\perp \text { up }}\right| \\
& \text { below: } \quad \text { below' } \stackrel{\text { def }}{=} \lambda A \cdot \lambda \mathbf{v} \cdot \operatorname{ext}(\mathbf{v}, A) \wedge c(-u p, \mathbf{v})>\left|\mathbf{v}_{\perp-u p}\right| \\
& \text { in front of: } \quad \text { in_front }{ }^{\prime} \stackrel{\text { def }}{=} \lambda A \cdot \lambda \mathbf{v} \cdot \operatorname{ext}(\mathbf{v}, A) \wedge c(\text { front }, \mathbf{v})>\left|\mathbf{v}_{\perp \text { front }}\right| \\
& \text { behind: } \quad \text { behind } \stackrel{\text { def }}{=} \lambda A . \lambda \mathbf{v} \cdot \operatorname{ext}(\mathbf{v}, A) \wedge c(- \text { front }, \mathbf{v})>\left|\mathbf{v}_{\perp-\text { front }}\right|
\end{aligned}
$$

The definition of beside' $\mathbf{e}^{\prime}$ is in terms of the absolute value of $c($ right, $\mathbf{v})$ :

$$
\text { (15) beside: beside } \stackrel{\text { def }}{=} \lambda A \cdot \lambda \mathbf{v} \cdot \operatorname{ext}(\mathbf{v}, A) \wedge \mid c(\text { right, } \mathbf{v})|>| \mathbf{v}_{\perp \text { right }} \mid
$$

The vertical symmetry of the regions generated by this definition is due to the fact that an equivalent definition is obtained when replacing right by -right. 


\subsection{Example: transitivity of 'between'}

The compositional mechanism and the lexical definitions above allow a correct analysis of many simple inferences. A central one is the transitive behaviour of between:

$A$ is between $B$ and $C$

$$
\begin{aligned}
& \mathrm{D} \text { is between } \mathrm{A} \text { and } \mathrm{B} \\
& \mathrm{D} \text { is between } \mathrm{B} \text { and } \mathrm{C}
\end{aligned}
$$

The following proposition specifies the conditions under which (16) holds in the proposed system. For convenience we refer to $A$ instead of $\operatorname{loc}(A)$, etc.

Proposition 5 a. If $A, B, C$ and $D$ are singletons $\{a\},\{b\},\{c\}$ and $\{d\}$, then the inference (16) generally holds. b. Otherwise, (16) holds with the additional assumption $\mathrm{D}$ is outside $\mathrm{C}$.

Proof: a. By definition if $A \subseteq \operatorname{co}(B \cup C) \backslash B \backslash C$ then $a \in[b, c] \backslash\{b\} \backslash\{c\}=(b, c)$. Similarly $d \in(a, b)$. Thus $d \in(b, c)$, or $D \subseteq \operatorname{co}(B \cup C) \backslash B \backslash C$.

b. Assume $A \subseteq \operatorname{co}(B \cup C) \backslash B \backslash C$. Thus $A \subseteq \operatorname{co}(B \cup C)$. By definition of co: $A \cup B \subseteq \operatorname{co}(B \cup C)$ and therefore $\operatorname{co}(A \cup B) \subseteq \operatorname{co}(B \cup C)$ (i).

Assume further $D \subseteq \operatorname{co}(A \cup B) \backslash A \backslash B$ (ii). $B y(i)$ and (ii): $D \subseteq \operatorname{co}(B \cup C)$. $D \cap B=\emptyset$ by (ii). $D \cap C=\emptyset$ by the proviso D is outside C. Concluding: $D \subseteq \operatorname{co}(B \cup C) \backslash B \backslash C$ as required.

Without the assumption $D$ is outside $C$ inference (16) does not necessarily follow, both intuitively and in our system. Consider figure $3 \mathrm{~b}$. Point $A$ may be taken (for instance, by a spectator located on line $l$ ) to be between rectangle $C$ and line $B$. Point $D$ is between $A$ and $B$. Nevertheless, the conclusion $D$ is between $B$ and $C$ is illegitimate.

\section{Denotational Properties of Locative Prepositions}

The study of various denotational properties of linguistic expressions is prominent in modeltheoretic semantics. This enterprise helps to reveal constraints on possible meanings ("semantic universals") and enables to classify semantic properties of expressions that affect grammaticality. The vector semantics of locative PPs has some non-trivial implications in both respects.

\subsection{Point monotonicity}

Let us call functions mapping sets of points to sets of vectors by the name prepositional functions. Since we are interested in the "pointal" behaviour of such functions let us concentrate on the end-points of vectors in the range of the function. For every prepositional function $P$ we denote the corresponding function $P^{e}$ from sets of points to sets of points as follows:

$$
P^{e} \stackrel{\text { def }}{=} \lambda A . \lambda \mathbf{p} . \exists \mathbf{v} \in P(A)[\mathbf{p}=e \text {-point }(\mathbf{v})]
$$


Definition 4 characterizes the monotonicity of a prepositional function on the set of points argument.

Definition 4 (point monotonicity) Let $P$ be a prepositional function and $X \subseteq$ $D_{p t}$.

1. $P$ is upward point-monotone over $X$ (PMON $\uparrow)$ iff $\forall A, B \in X\left[A \subseteq B \rightarrow P^{e}(A) \subseteq P^{e}(B)\right]$.

2. $P$ is downward point-monotone over $X(\mathrm{PMON} \downarrow)$ iff $\forall A, B \in X\left[A \subseteq B \rightarrow P^{e}(B) \subseteq P^{e}(A)\right]$.

We specify point-monotonicity of prepositions according to the behaviour of their denotation over the domain $X$ of topologically simple regions. Intuitively, pointmonotonicity corresponds to truth preservation under enlargement/diminution of the reference object. For instance, knowing that Paris is in France, we may conclude that both (17a) and (17b) hold.

(17) a. The house is in Paris $\Rightarrow$ The house is in France

b. The house is outside France $\Rightarrow$ The house is outside Paris

This suggests that in and outside are $\mathrm{PMON} \uparrow$ and $\mathrm{PMON} \downarrow$ prepositions respectively. The functions inside' ${ }^{\prime}$ and outside' verify this over the domain of topologically simple regions and establish entailments (17a-b). Generally, the inference schemes below characterize the point-monotonicity of a preposition $\mathrm{P}$.

$$
\mathrm{P} \in \mathrm{PMON} \uparrow: \quad \mathrm{P} \in \mathrm{PMON} \downarrow:
$$

\begin{tabular}{ll}
$\mathrm{A}$ is inside $\mathrm{B}$ & $\mathrm{A}$ is inside $\mathrm{B}$ \\
$\mathrm{C}$ is $\mathrm{P} \mathrm{A}$ & $\mathrm{C}$ is P B \\
\cline { 1 - 1 } $\mathrm{C}$ is P B & $\mathrm{C}$ is P A
\end{tabular}

Other prepositions besides inside and outside are not point-monotone. Consider the preposition above for example. In figure $3 \mathrm{c}$ the sentence the bird is above the house is true, assuming that the bird is $\mathbf{b}$ and the house is $H$. However, the same sentence is false when we consider a much smaller house $h$ or a much larger house $H^{\prime}$. Hence, above is neither downward nor upward point-monotone. We propose the following universal.

Universal 1 Only inside' and outside' are possible $\mathrm{PMON} \uparrow$ and $\mathrm{PMON} \downarrow$ denotations, respectively, for simple locative prepositions in natural language.

Consider the expression far from in English. The corresponding prepositional function is PMON $\downarrow$ but different than the meaning of outside. Universal 1 claims that this function is not a possible denotation for single word locative prepositions in any natural language. Note that far from in English is neither simple nor evidently locative. It is a compound (not necessarily constituent), derived from the directional preposition from. Unlike far from, a non-PMON preposition like near is realized as a single lexical morpheme in many languages.

Another example for meanings of prepositions that are ruled out by universal 1 are the following functions, sensitive to the diameter of the reference object. Recall the diameter of a set $A$ is the supremum of the set $\{\operatorname{dist}(x, y): x, y \in A\}$. 


$$
\begin{aligned}
& \text { outside }_{\text {diam }<10}^{\prime}(A)(\mathbf{v}) \text { iff } \operatorname{outside}^{\prime}(A)(\mathbf{v}) \text { and } \operatorname{diam}(A)<10 \\
& \text { inside }_{\text {diam }>10}^{\prime}(A)(\mathbf{v}) \text { iff } \operatorname{inside}^{\prime}(A)(\mathbf{v}) \text { and } \operatorname{diam}(A)>10
\end{aligned}
$$

These functions are PMON $\downarrow$ and $\mathrm{PMON} \uparrow$ respectively, but different than outside' and inside'.

The prepositions outside and inside are special in another respect. By proposition 2 , over topologically simple objects outside ${ }^{e}$ and inside $e^{e}$ coincide with set complementation and identity respectively: for every topologically simple $A \subseteq D_{p}$ : outside $^{e}(A)=\bar{A}$ and $\operatorname{inside}^{e}(A)=A$. For all other natural language prepositions $P^{e}$ is not set-theoretically definable without further assumptions on $D_{p}$ (e.g. the above space ontology). Moreover, we propose:

Universal 2 For every prepositional function $P$ referred to by a locative preposition in natural language, for every topologically simple region $A: P^{e}(A) \subseteq \bar{A}$ or $P^{e}(A)=A$.

In other words, for every preposition $\mathrm{P}$ either (19a) or (19b) must hold.

(19) a. $A$ is $P B \Rightarrow A$ is outside $B \quad$ b. $A$ is $P B \Leftrightarrow A$ is inside $B$

Thus, the meaning of inside is the only preposition meaning that does not entail outside. This eliminates prepositions that entail inside but are not entailed by it. For instance, an imaginable preposition *nearin satisfies $A$ is nearin $B$ iff $A$ is inside $\mathrm{B}$ and $\mathrm{A}$ is close to B's edge. This preposition does not satisfy universal 2 and is unexpected to occur in any natural language. Also prepositions that entail neither inside nor outside are eliminated. For instance, a preposition equivalent to either near or else inside is ruled out. These predictions are correct as far as we know.

Most prepositions are not point-monotone. A weaker property, however, holds of all prepositions:

Definition 5 (point continuity) Let $P$ be a prepositional function and $X \subseteq D_{p \ell}$. $P$ is point continuous over $X$ (PCON) iff $\forall A, B, C \in X[A \subseteq B \subseteq C \rightarrow$ $\left.P^{e}(A) \cap P^{e}(C) \subseteq P^{e}(B)\right]$.

Point monotonicity entails point continuity. Intuitively, a point continuity test is one that can be called the "Babushka inference". Suppose that Babushka 1 is inside Babushka 2, which is inside Babushka 3. The following inference holds of the PCON preposition beside:

(20) The ball is beside Babushka 1 and beside Babushka 3

$\Rightarrow$ The ball is beside Babushka 2

We claim that the same holds of all prepositions.

Universal 3 All prepositions in natural language are point continuous.

This universal is similar to the continuity universal of Thijsse (1983) on "simple" NPs (universal U6 of Barwise and Cooper (1981)). Thus, continuity holds of determiners and prepositions alike. Universal 3 rules out an imaginable preposition *aboven that satisfies $\mathrm{A}$ is *aboven $\mathrm{B}$ iff $\mathrm{A}$ is an even number of meters above $\mathrm{B}$. Another example for a non-PCON prepositional function is the following:

outside $_{\text {even (diam) }}^{\prime}(A)(\mathbf{v})$ iff outside $(A)(\mathbf{v})$ and $\operatorname{diam}(A)$ is an even natural number 


\subsection{Vector monotonicity}

Prepositional functions are relations between sets of points and vectors. The following partial order ${ }^{9}$ on $D_{v}$ allows us to examine their monotonicity with respect to the vector argument.

Definition 6 (vector order) For all $\mathbf{v}, \mathbf{w} \in D_{v}: \mathbf{v} \leq \mathbf{w}$ iff there is $s \geq 1$ in $\mathbf{R}$ s.t. $\mathbf{w}=s \mathbf{v}$.

Intuitively, $\leq$ is a relation of lengthening over non-zero vectors $\mathbf{v}, \mathbf{w}$ that have the same start point. For such vectors $\mathbf{v} \leq \mathbf{w}$ iff $\mathbf{v}$ and $\mathbf{w}$ point in the same direction and $|\mathbf{v}| \leq|\mathbf{w}|$.

Definition 7 (vector monotonicity) Let $P$ be a prepositional function and $X \subseteq$ $D_{p t}$.

1. $P$ is upward vector-monotone over $X(\mathrm{VMON} \uparrow)$ iff $\forall A \in X \forall \mathbf{u}, \mathbf{v} \in D_{v}[\mathbf{u} \leq \mathbf{v} \rightarrow(P(A)(\mathbf{u}) \rightarrow P(A)(\mathbf{v}))]$.

2. $P$ is downward vector-monotone over $X(\mathrm{VMON} \downarrow)$ iff $\forall A \in X \forall \mathbf{u}, \mathbf{v} \in D_{v}[\mathbf{u} \leq \mathbf{v} \rightarrow(P(A)(\mathbf{v}) \rightarrow P(A)(\mathbf{u}))]$.

Vector monotonicity corresponds to truth preservation when the located object gets further from/closer to the reference object. For instance, when Mary is between the tree and the house, both (21a) and (21b) hold. This suggests that behind is both $\mathrm{VMON} \uparrow$ and $\mathrm{VMON} \downarrow$.

(21) a. Mary is behind the house $\Rightarrow$ The tree is behind the house

b. The tree is behind the house $\Rightarrow$ Mary is behind the house

Generally, the following inferences indicate vector monotonicity of a preposition $\mathrm{P}$.

$\mathrm{P} \in \mathrm{VMON} \uparrow:$

An example for a non-VMON $\uparrow$ preposition is near: if $\mathrm{A}$ is near $\mathrm{B}$ and gets further from it, at a certain point it will no longer be near B. More examples are given below.

(23) VMON $\uparrow$ : in front of, behind, above, below, beside, ${ }^{10}$ outside not $\mathrm{VMON} \uparrow:$ near, on, at, in/inside, between ${ }^{11}$

Examples for prepositions that are not VMON $\downarrow$ are harder to find. The expression far from could be considered a possible candidate. However, for similar reasons to the aforementioned, we do not take this as a counter-example to the following universal from Zwarts (1995).

Universal 4 All simple locative prepositions in natural language are $\mathrm{VMON} \downarrow$. 
Also the artificial preposition *not_close_to_and_not_far_from, with the obvious semantics, is not VMON $\downarrow$. Unlike far from it is not $\mathrm{VMON} \uparrow$ either.

$\mathrm{V}$-monotonicity is relevant to the grammaticality of PP modification. Consider the contrast between (24) and (25) vis-à-vis the $\mathrm{VMON} \uparrow$ properties in (23).

(24) a. two meters in front of/behind/above/below the car

b. ?two meters beside the car

c. two kilometers outside the village

(25) a. *two meters near/on/at the house

b. *two meters in/inside the house

c. *two meters between the two houses

Observing this compatibility, Zwarts (1995) proposes that modification of a PP using a measure phrase modifier is legitimate if and only if the PP is headed by a $\mathrm{VMON} \uparrow$ preposition. Consider, however, sentence (26) in contrast to (25b).

(26) The nail is $10 \mathrm{~cm}$ inside the wall.

Modification in (26) is allowed although inside is not VMON $\uparrow$. Nevertheless, there is a possible contrast between the eigenspaces that are likely to correspond to the reference objects in the the two cases. We speculate that a wall as in (26) can be conceived of as an "unbounded" object: from the point of view of a person on one of its sides, a wall might have been unbounded on the other side. This is unlikely to be the case with the eigenspace of the house in (25b). Thus, we propose that the relevant property is not vector monotonicity of the preposition, but rather monotonicity of the set of vectors being modified. This is stated as follows.

(27) Modification Generalization: A structure [P NP] can be modified by a measure phrase iff its denotation $W_{v t}$ is $\mathrm{VMON} \uparrow: \forall \mathbf{u} \in W \forall \mathbf{v} \in D_{v}[\mathbf{u} \leq \mathbf{v} \rightarrow$ $\mathbf{v} \in W]$.

If $\mathrm{P}$ is a $\mathrm{VMON} \uparrow$ preposition then, according to (27), modification should be possible for every possible reference object. This accounts for the acceptability of the cases in (24). For non-VMON $\uparrow$ prepositions and bounded reference objects, (27) expects modification to be impossible, which accounts for (25). However, with inside and an unbounded reference object as in (26) we still have a $\mathrm{VMON} \uparrow$ set of vectors, although the preposition itself is not VMON $\uparrow$. Thus (27) describes also the acceptability of (26). But what can be an explanation for the rule of thumb in (27)? Assume that measure phrase modification is possible iff every non-trivial measure phrase maps $W$ to a non-empty set. ${ }^{12}$ By universal $4, W$ must be VMON $\downarrow$. Thus, it is sufficient to require that $W$ is $\mathrm{VMON} \uparrow$ (and non-empty) in order to guarantee this non-emptiness condition. We propose that this semantic requirement is grammaticized to affect the acceptability of modified PPs. In other words, (27) plus universal 4 make sure that non-trivial measure phrases are semantically effective in the modification process of the PP. For instance, a PP like $* 5000 \mathrm{~km}$ near the house is not simply ungrammatical, but also semantically absurd if its meaning is computed. See Barwise and Cooper (1981:p.183) for a similar reasoning about relations between semantic properties of determiners and grammaticality of there sentences. 


\subsection{Preposition conservativity}

In the proposed system there is an important relation between the two arguments of locative prepositions. Consider the following property.

Definition 8 (preposition conservativity) A prepositional function $P$ is called conservative $\left(\mathrm{CONS}_{P}\right)$ iff $\forall A \forall \mathbf{v}[P(A)(\mathbf{v}) \rightarrow$ s-point $(\mathbf{v}) \in A]$.

A preposition is called conservative if $\mathrm{f}$ its denotation is conservative in every model. In a way that is similar to determiner conservativity, ${ }^{13}$ when $P$ is a conservative prepositional function the argument $A$ restricts the set of possible elements in $P(A)$ : only vectors whose start-point is in $A$ can be in $P(A)$. We propose:

Universal 5 All natural language locative prepositions are conservative.

This claim is related to $^{14}$ the following inference.

(28) $\mathrm{A}$ is $n$ meters $\mathrm{P} \mathrm{B} \Rightarrow$ The distance between $\mathrm{A}$ and $\mathrm{B}$ is $n$ meters

All the prepositions discussed above are conservative. (28) holds of all these prepositions when modification is grammatical. Consider, however, an artificial nonconservative preposition *behose (=behind something close to), whose denotation is naturally defined as follows:

$\operatorname{behose}^{\prime}(A)(\mathbf{v})$ iff $\exists B_{p t}\left[\right.$ behind $^{\prime}(B)(\mathbf{v}) \wedge \forall \mathbf{p} \in B \exists \mathbf{w} \in \operatorname{near}^{\prime}(A)$

$$
[\mathbf{p}=e \text {-point }(\mathbf{w})]]
$$

This is a non-conservative preposition, which is $\mathrm{VMON} \uparrow$ and $\mathrm{VMON} \downarrow$ like behind Thus, modification by measure phrases should be syntactically possible. However, (28) does not hold of this preposition. An object A can be 3 meters behind something close to $\mathrm{B}$ without the distance between $\mathrm{A}$ and $\mathrm{B}$ being 3 meters. Universal 5 expects prepositions equivalent to ${ }^{*}$ behose to exist in no natural language. Below we mention another such non-preposition *inose, similarly defined as equivalent to inside something close to.

Like conservativity in the determiner domain, also preposition conservativity imposes a reduction in the number of possible denotations for lexical prepositions. To get an impression of that, let us tentatively assume that $D_{p} \subset V$ is finite and $\left|D_{p}\right|=n$, and that $D_{v}=\left\{\langle w, v\rangle \in V \times V: w \in D_{p}\right.$ and $\left.w+v \in D_{p}\right\}$. That is, $D_{v}$ is the set of vectors over the points in $D_{p}$. Let us denote $P R E P=D_{(p t)(v t)}$.

Proposition $6|P R E P|=2^{2^{n} \cdot n^{2}} \quad\left|C O N S_{P}\right|=2^{2^{n-1} \cdot n^{2}}$

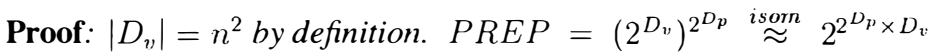

Thus, $|P R E P|=2^{2^{n} \cdot n^{2}}$.

$\left.C O N S_{P} \stackrel{\text { isom }}{\approx} 2^{\left\{\langle A, \mathbf{w}\rangle \in 2^{D_{p}} \times D_{v}: s-p o i n t\right.}(\mathbf{w}) \in A\right\}$

Let $A \in 2^{D_{p}}$ be of cardinality $i$. Thus, the set $\left\{\mathbf{w} \in D_{v}: s\right.$-point $\left.(\mathbf{w}) \in A\right\}$ (the vectors in $D_{v}$ with start point in $\left.A\right)$ is of cardinality $i \cdot n$. Therefore we get:

$\left|\left\{\langle A, \mathbf{w}\rangle \in 2^{D_{p}} \times D_{v}: \operatorname{s-point}(\mathbf{w}) \in A\right\}\right|$ 


$$
\begin{aligned}
& =\sum_{i=0}^{n}\left(\begin{array}{c}
n \\
i
\end{array}\right) \cdot i \cdot n \quad\left(\left(\begin{array}{c}
n \\
i
\end{array}\right) \text {-the number of subsets of } D_{p} \text { of cardinality } i\right) \\
& =n \sum_{i=0}^{n} \frac{n !}{i ! \cdot(n-i) !} \cdot i=n^{2} \sum_{i=0}^{n-1}\left(\begin{array}{c}
n-1 \\
i
\end{array}\right)=2^{n-1} \cdot n^{2}
\end{aligned}
$$

Compare this to the reduction D-conservativity imposes on the number of determiner denotations (van Benthem (1984)):

(29) For $\left|D_{e}\right|=n,\left|D_{(e t)((e t) t)}\right|=|D E T|=2^{4^{n}}$ whereas $\left|C O N S_{D}\right|=2^{3^{n}}$

P-conservativity is a weaker constraint than D-conservativity. It reduces the second exponent while the latter reduces the second base. The difference is clear from the following table, calculating the number of (conservative) Ds and Ps for $n=2,3,4$ :

$\begin{array}{ccccc}n & |D E T| & \left|C O N S_{D}\right| & |P R E P| & \left|C O N S_{P}\right| . \\ 2 & 65,536 & 512 & 65,536 & 256 \\ 3 & \sim 1.84 \times 10^{19} & \sim 1.34 \times 10^{8} & \sim 4.72 \times 10^{21} & \sim 6.87 \times 10^{10} \\ 4 & \sim 1.16 \times 10^{77} & \sim 2.42 \times 10^{24} & \sim 1.16 \times 10^{77} & \sim 3.4 \times 10^{38}\end{array}$

Already in a domain with 4 elements, D-conservativity eliminates more denotations than P-conservativity, although in this case the total number of preposition and determiner denotations is the same. Note, however, that $\mathrm{CONS}_{P}$ is not the strongest restriction we can obtain when also spatial properties are considered. On its own, it is not sufficient to guarantee the sound inference (28). In fact, if $P(A)(\mathbf{v})$ holds then $s$-point $(\mathbf{v})$ is not just any point in $A$, but rather a member of $A$ 's boundary that is closest to $e$-point $(\mathbf{v})$. Measuring the reduction in possible prepositions that this spatial property imposes is much harder than with the set-theoretical property $\mathrm{CONS}_{P}$, so we must leave this question aside for the time being.

Recapitulation Note that the universals 1-5 are independent. This can be shown by verifying that each of the artificial prepositions outside diam $<10_{10} /$ inside $_{\text {diam }>10}$, nearin, outside even(diam), not_close_to_and_not_far_from, and inose is ruled out only by the corresponding universal.

\section{Appendix: some useful definitions}

A vector space over the field of real numbers $\mathbf{R}$ is a quadruple $\langle V, 0,+, \cdot\rangle$ s.t. $V$ is a set, $0 \in V$ (the zero vector) and the functions $+:(V \times V) \rightarrow V$ (vector addition) and $\cdot(\mathbf{R} \times V) \rightarrow V$ (scalar multiplication) satisfy for all $u, v, u \in V$ and $s, r \in \mathbf{R}$ :

1. $(u+v)+w=u+(v+w)$

2. $0+v=v+0=v$

3. There is an element $-v \in V$ s.t. $v+(-v)=0$

4. $u+v=v+u$

5. $s(u+v)=s u+s v$ 
6. $(s+r) v=s v+r v$

7. $(s r) v=s(r v)$

8. $1 \mathrm{v}=\mathrm{v}(1$ is the unit element of $\mathbf{R})$

A scalar product over a vector space $V$ is a function $f:(V \times V) \rightarrow \mathbf{R}$ that satisfies for all $u, v, w \in V, s \in \mathbf{R}$ :

1. $f(v, w)=f(w, v)$

2. $f(u, v+w)=f(u, v)+f(u, w)$

3. $f(s v, w)=s f(v, w)=f(v, s w)$

A scalar product is called positive iff for every $v \in V: f(v, v) \geq 0$ and for every $v \in V \backslash\{0\}: f(v, v)>0$. For a positive scalar product $f$ the norm of a vector $v \in V$ is denoted $|v|=\sqrt{f(v, v)}$. We say that two vectors $v, w \in V$ are orthogonal (perpendicular) and denote $v \perp w$ iff $f(v, w)=0$. For any vector $w \in V$ we call $\perp w \stackrel{\text { def }}{=}\{v \in V: v \perp w\}$ the orthogonal complement of $w$.

For $v, w$ in a space $V$, the line segment $[v, w]$ is the set $\{s v+(1-s) w \because 0 \leq$ $s \leq 1\}$. A set $A \subseteq V$ is convex iff for all $v, w \in A:[v, w] \subseteq A$. The convex hull of $A$ is the smallest convex subset of $V$ containing $A$.

For any set $X$ a metric for $X$ is a non-negative function $d:(X \times X) \rightarrow \mathbf{R}^{+}$ that satisfies for all $x, y, z \in X$ :

1. $d(x, y)=d(y, x)$

2. $d(x, y)+d(y, z) \geq d(x, z)$

3. $d(x, y)=0$ iff $x=y$

Note that the function $\operatorname{dist}(v, w)=|v-w|$ is a metric for $V$. A neighborhood in $X$ of a point $x \in X$ is defined by $U_{X}(x, \delta)=\{y \in X: d(x, y)<\delta\}$. A set $A \subseteq X$ is open in $X$ iff for every $x \in A$ there is $\delta>0$ s.t. $U_{X}(x, \delta) \subseteq A . A \subseteq X$ is closed in $X$ iff $X \backslash A$ is open in $X$. The interior of $A \subseteq X$ is the union of all open subsets in $X$ contained in $A$. The boundary of $A$ is the set of points in $X$ interior neither to $A$ nor to $X \backslash A$. The diameter of $A$ is the supremum of the set $\{d(x, y): x \in A$ and $y \in A\}$. A set $A$ is bounded iff its diameter is finite. We do not define compact sets, rather only mention the Heine-Borel-Lesbegue theorem (see Kelley (1961:p.144)): a subset of an Euclidean n-space $\mathbf{R}^{n}$ is compact iff it is closed and bounded.

\section{Endnotes}

* The part of the first author was supported by the Foundation for Language, Speech and Logic, which is funded by the Netherlands Organization for Scientific Research, NWO (grant 300-171-033). The part of the second author was subsidized by an NWO grant and a grant of the Dutch school in Linguistics, LOT. We are grateful to Johan van Benthem and Ed Keenan for their remarks on this work.

1. Lang (1977) and Kelley (1961) are two of the many introductory textbooks in these fields.

2. Symmetry with near is incomplete. For instance, reasonably due to pragmatic 
factors, the bicycle is near the house is OK whereas the house is near the bicycle is a weird sentence. Similar asymmetries hold for some. Compare for instance some people are politicians with some politicians are people.

3. Of course, in English there are non-spatial prepositions (e.g. before, of) and most spatial prepositions can also be used for expressing non-spatial statements (e.g. Mary arrived on Tuesday).

4. We concentrate only on measure modifiers like ten meters and not on direction modifiers like diagonally. In addition, sentences like (7b) with measure phrases are arguably ambiguous. One reading requires that the distance between the tree and the house is ten meters. Another reading states that the distance to the plane orthogonal to the frontal axis of the house (see below) is ten meters. We deal only with the first possibility. Extending our approach to the other cases is unproblematic but requires some complications that are unnecessary for our objectives.

5. This is a convenience rather than a necessity. A notational variant is to assume a point ontology together with a metric and treat prepositions as functions from sets of points to sets of ordered pairs of points, so vectors are indirectly represented. In our strategy the metric function is implicitly supplied by the given scalar product.

6. Arguably, this condition is too strong because even one vector satisfying it can make (7a) true. We believe, however, that this is due to pragmatic effects in the determination of loc: speakers' spatial conception of an object is often significantly different than its physical shape. Reasonably, even large objects like trees and houses can be represented as volume-less points. In such cases universal quantification as in (9) is indeed equivalent to existential quantification, as $l o c$ (the_tree') is a singleton. Changing the definition of $l o c^{-}$so that (9) expresses existential quantification would be more problematic. For instance, (3a) would become true even if only a tip of one leaf of the tree is outside the house.

7. We do not distinguish between in and inside despite some distributional differences between them (e.g. in the air vs. *inside the air). We also ignore the intricate meaning aspects of in discussed in Herskovits (1986) and Vandeloise (1991). For example, why do we say in the field but not in the prairie? Why don't we usually use the expression in the bowl when a bowl is upside down? Henceforth we put these questions aside, focusing on the more general semantic issues.

8. Again, we are only interested in the general idea and ignore questions concerning the determination of this $r_{1}$, its dependence on the size of the reference object, etcetera. See Crangle and Suppes (1989) for discussion. Also the differences between on and at (like on the desk vs. at the desk) are ignored. We refer to the literature mentioned earlier.

9. Recall a partial order on $D_{v}$ is a relation $\leq$ that satisfies for all $\mathbf{u}, \mathbf{v}, \mathbf{w} \in D_{v}$ : $\mathbf{u} \leq \mathbf{u}$ (reflexivity), if $\mathbf{u} \leq \mathbf{v}$ and $\mathbf{v} \leq \mathbf{u}$ then $\mathbf{u}=\mathbf{v}$ (antisymmetry), if $\mathbf{u} \leq \mathbf{v}$ and $\mathbf{v} \leq \mathbf{w}$ then $\mathbf{u} \leq \mathbf{w}$ (transitivity).

10. Whether beside should really be upward monotone as our definition implies is not completely clear. It may be that in English this preposition carries an element of proximity, which would put it in the other category.

11. Given the special type of this preposition, its monotonicity is determined by a trivial variation on definition 7 . 
12. A measure phrase like less than $O m$ is trivial as it denotes a constant function returning the empty set.

13. Recall a determiner $D$ is conservative iff $B \in D(A) \leftrightarrow B \cap A \in D(A)$ for all $A$ and $B$.

14. (28) is sufficient, but not necessary, for conservativity to hold. See below.

\section{References}

Barwise, J. and Cooper, R. (1981). Generalized quantifiers and natural language. Linguistics and Philosophy, 4:159-219.

Crangle, C. and Suppes, P. (1989). Geometrical semantics for spatial prepositions. Midwest Studies in Philosophy, 14:399-422.

Herskovits, A. (1986). Language and Spatial Cognition: an interdisciplinary study. of the prepositions in English. Cambridge University Press, Cambridge.

Keenan, E. (1996). The semantics of determiners. In Lappin, S., editor, The Handbook of Contemporary Semantic Theory. Blackwell.

Kelley, J. L. (1961). General Topology. Van Nostrand, Princeton, New Jersey.

Lang, S. (1977). Linear Algebra. Addison-Wesley, Reading, Massachusetts, second edition.

Thijsse, E. (1983). On some proposed universals of natural language. In ter Meulen, A., editor, Studies in Modeltheoretic Semantics. Foris, Dordrecht.

van Benthem, J. (1984). Questions about quantificrs. Journal of Symbolic Logic, $49: 443-466$.

Vandeloise, C. (1991). Spatial Prepositions. Chicago University Press, Chicago.

Wall, C. T. C. (1972). A Geometric Introduction to Topology. Addison-Wesley, Reading, Massachusetts.

Wunderlich, D. (1991). How do prepositional phrases fit into compositional syntax and semantics. Linguistics, 29:591-621.

Zwarts, J. (1995). The semantics of relative position. In Proceedings of Semantics and Linguistic Theory, SALT5. A revised version is to appear in Journal of Semantics.

Zwarts: Joliotplaats 666, 3069 TS Rotterdam, The Netherlands.

Winter: U.i.L. OTS, Trans 10, 3512 JK Utrecht, The Netherlands.

E-mail: yoad.winter@let.ruu.nl

WWW: http://wwwots.let.rut.nl/cgi-bin/staff?winter 